\title{
Research Paper \\ The Effect of Exercise Timing on Elevated Postprandial Glucose in Women With Obesity
}

\author{
Fateme Dehghani Yunarti ${ }^{1}\left(\mathbb{C},{ }^{*}\right.$ Vazgen Minasian $^{1}$ ()
}

1. Department of Exercise Physiology, Faculty of Sport Sciences, University of Isfahan, Isfahan, Iran.

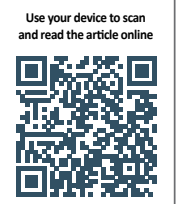

cittation: Dehghani Yunarti F, Minasian V. [The Effect of Exercise Timing on Elevated Postprandial Glucose in Women With Obesity (Persian)]. Journal of Arak University of Medical Sciences (JAMS). 2021; 24(3):334-347. https://doi.org/10.32598/ JAMS.24.3.5445.2

doi https://doi.org/10.32598/JAMS.24.3.5445.2

Key words:

Exercise, Postprandial

Glucose, Obesity

\section{A B STRACT}

Article Info:

Received: 31 Oct 2020

Accepted: 15 May 2021

Available Online: 01 Aug 2021

\section{Extended Abstract}

\section{Introduction}

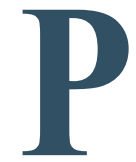

ostprandial Hypertension (PPG) is one of the significant risk factors for many metabolic-cardiac disorders in diabetic $[1,2]$ and non-diabetic $[3,4]$ patients. For many metabolic disorders, including obesity and Type 2 Diabetes (T2D), the main focus of treatment is to reduce postprandial hyperglycemia. Endothelial dysfunction increased oxidative stress, and the risk of cardiovascular disease have been reported with increased PPG [5-7]. Regular exercise can be a good strategy for treating many metabolic disorders [8] and is a potential safety and appropriate strategy for reducing postprandial blood hyperglycemia due to repetitive muscle contractions $[9,10]$. These changes may be due to the acute increase in skeletal muscle insulin sensitivity for 24-48 hours after each exercise [11].

\footnotetext{
* Corresponding Author:

Vazgen Minasian, PhD.

Address: Department of Exercise Physiology, Faculty of Sport Sciences, University of Isfahan, Isfahan, Iran.

Tel: +98 (913) 2020396

E-mail: v.minasian@spr.ui.ac.ir
} 
Table 1. Some measured variables in participants

\begin{tabular}{cc}
\hline Variables & Mean \pm SD \\
\hline Age $(\mathrm{y})$ & $40.41 \pm 3.09$ \\
Weight $(\mathrm{kg})$ & 86.67 .26 \\
Height $(\mathrm{cm})$ & $161.40 \pm 3.56$ \\
Body mass index $\left(\mathrm{kg} / \mathrm{m}^{2}\right)$ & $33.22 \pm 2.20$ \\
Body Fat Percentage (\%) & $35.70 \pm 3.36$ \\
Basal Metabolic Rate (Kcal) & $1574.05 \pm 68.94$ \\
Aerobic Endurance $(\mathrm{ml} / \mathrm{kg}-1 / \mathrm{min}-1)$ & $25.60 \pm 1.89$ \\
\hline
\end{tabular}

Therefore, this study aimed to investigate the effect of exercise training scheduling (before meals, after meals, and periodically throughout the day) on increased postprandial glucose levels in middle-aged obese women.

\section{Materials and Methods}

Ten women with obesity (Mean \pm SD age $=40.41 \pm 3.97$ years; weight $=86.66 \pm 7.26 \mathrm{~kg}$; and BMI $=33.22 \pm 2.20 \mathrm{~kg}$ / $\mathrm{m}^{2}$ ) participated in a crossover design. The interventions consist of 1) postprandial aerobic exercise, 2) pre-prandial aerobic exercise, 3) brief periodic exercise, and 4) control performed in 4 full days with 5 days rest between each train- ing session. Pre- and postprandial exercise included $30 \mathrm{~min}$ of moderate-intensity walking on the treadmill before and after each main meal (1 min of exercise $-30 \mathrm{sec}$ rest). Brief periodic exercise had three one-minute of activity every 30 min for 20 times during the day. The twelve-hour continuous glucose monitoring and two-hour postprandial glucose levels were measured to examine changes in blood glucose levels. The exercise interventions were performed randomly; all participants ate the same meals the day before the intervention. After 10 hours of fasting, they referred to the laboratory to take blood samples. The subjects' glucose levels were measured using the Iranian Pars test kit. The control group had daily activities without exercising, and participants' glucose levels were measured at specific periods.

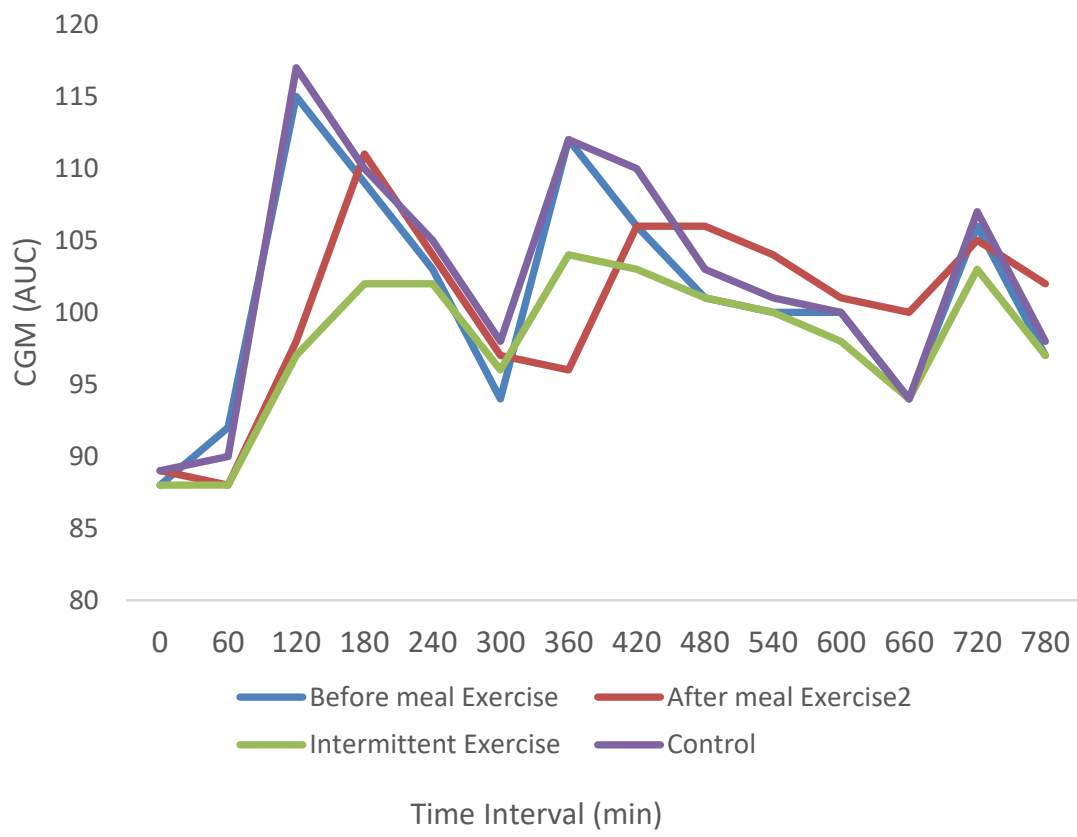

Figure 1. Changes in CGM in intervention groups 


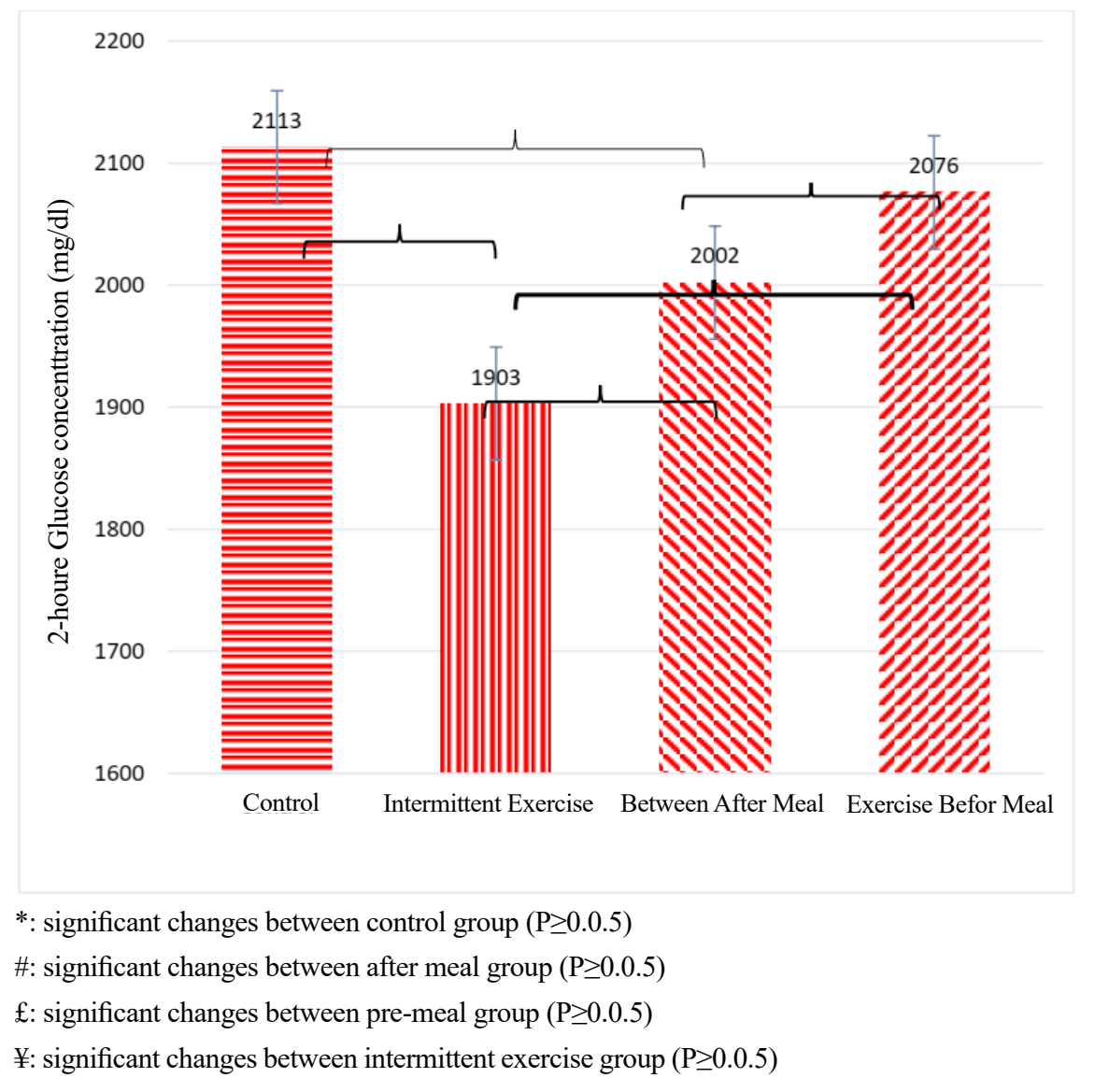

Figure 2. Two-hour gluccose changes in experimental groupe

\section{Results}

Descriptive characteristics of the participants are presented in Table 1. The results of data analysis showed that short-term intermittent activities led to a significant reduction in CGM compared to the control group and exercise before meals $(\sim 5 \%, \mathrm{P}=0.001)$. On the other hand, there was a significant difference between changes in CGM levels of exercise before and after meals $(\mathrm{P}<0.05)$ (Figure 1). In addition, the results showed that post-meal exercise and intermittent exercise resulted in a further decrease in PPG compared to the control group ( 6 and $~ 10 \%$, respectively) $(\mathrm{P}=0.01)$. In contrast, a decrease in PPG in the pre-meal exercise was insignificant compared to the control group $(\mathrm{P}>0.05)$. Also, intermittent exercise training was more effective in reducing PPG levels ( $\sim 9 \%$ vs $5 \%$, respectively) compared to exercise interventions before and after meals $(\mathrm{P}=0.004)$. Post-meal exercise also had more significant effects on post-meal glucose-lowering than pre-meal exercise $(4 \%)(\mathrm{P}=0.01)$ (Figure 2).

\section{Discussion and Conclusion}

The exercise after a meal led to an improvement in PPG levels. It has already been reported that exercising after a meal (45 min) lowers blood glucose and insulin levels in patients with diabetes. One of the mechanisms affecting the reduction of blood glucose is probably more skeletal muscle metabolism involved in the activity [34]. The effects of exercise after a meal may be due to the regulatory impact of exercise on glucose metabolism in the liver and skeletal muscle, maintained in obese individuals for 2 hours after exercise training. In contrast, the pre-meal exercise did not significantly affect PPG and CGM in women with obesity.

Previous studies indicated the effectiveness of intense exercise training and high lactate threshold before a meal PPG level. Still, on the other hand, due to the high intensity of exercise in overweight and obese people, they need much more motivation [41, 42]. Furthermore, the intermittent periodic exercise had significant effects on the improvement of CGM and PPG, which were more than the effects of exercise before and after meals. In this regard, recent studies 
have investigated the effect of walking on PPG levels in fasting status in obese and healthy individuals, which reduces CGM by 5 min of activity every 20 min for 5 hours $[34,36]$. Also, previous studies reported that different short-term intermittent exercises could reduce post-meal CGM levels [16, 25, 38].

Few studies have directly compared the glycemic responses between pre-and post-diet exercise interventions, and more research is needed in the future. One of the limitations of this study was the small sample size and the impossibility of studying 24-hour blood glucose levels using laboratory methods.

\section{Ethical Considerations}

\section{Compliance with ethical guidelines}

This study was approved by the Institutional Review Board of the University of Isfahan (Ethics Code: IR.UI. REC.1397.119) and conducted in agreement with the ethical principles for biomedical research involving human subjects outlined in the declaration of Helsinki.

\section{Funding}

This study was extracted from the MSc thesis of the first author at the Department of Exercise Physiology, Faculty of Sport Sciences, University of Isfahan, Isfahan. Also, this study was supported by the Chancellor for Research and Technology of the University of Isfahan for postgraduate students.

\section{Authors' contributions}

Both authors equally contributed to preparing this article.

\section{Conflicts of interest}

The authors declare no conflict of interest. 
This Page Intentionally Left Blank 


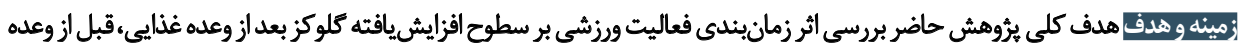

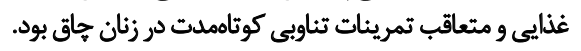

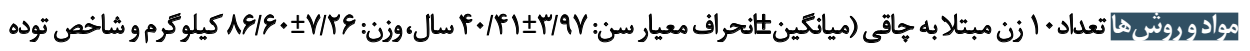

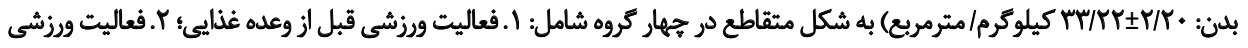

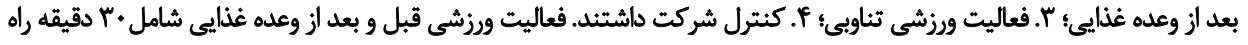

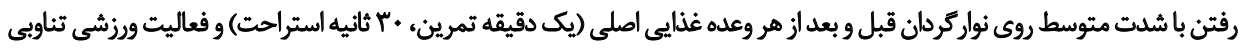

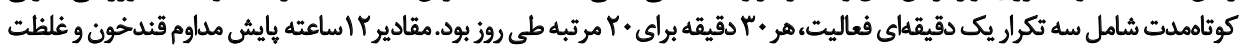

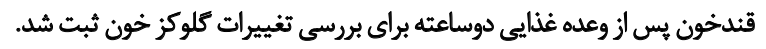

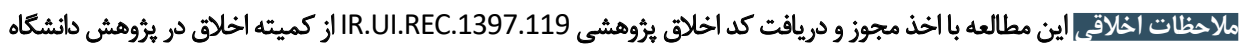

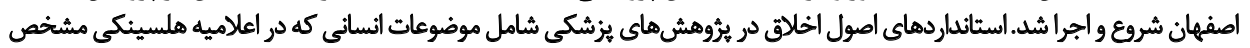

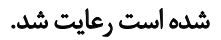

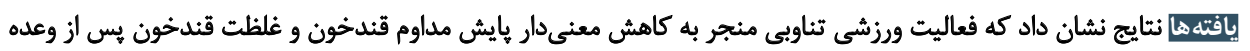

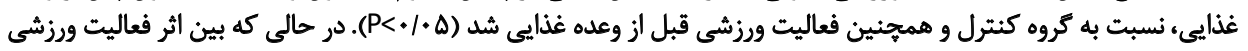

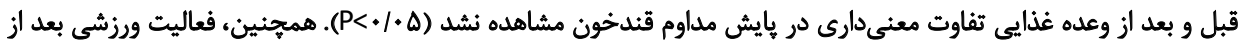

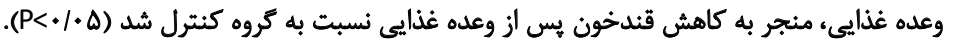

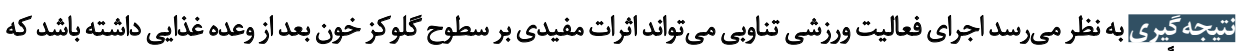

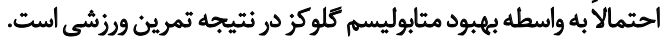

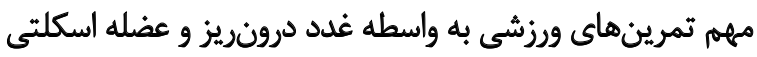

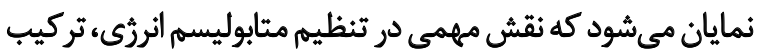

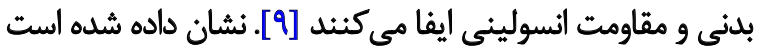

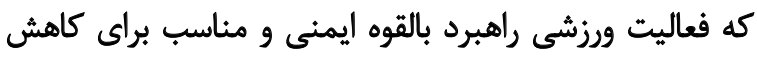

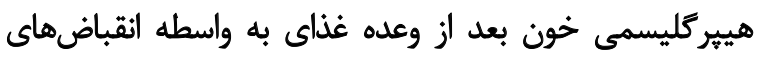

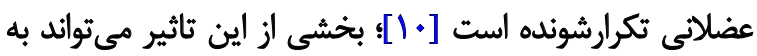

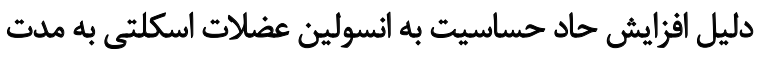

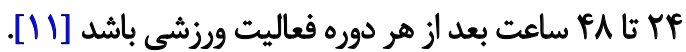

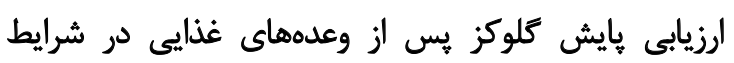

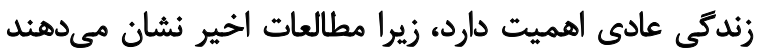

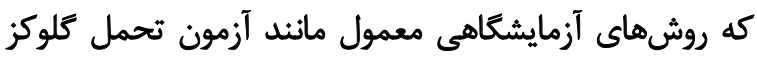

غلظت زياد قندخون يس از وعده غذايى يكيى از عوامل خطرزاي

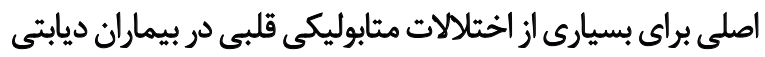

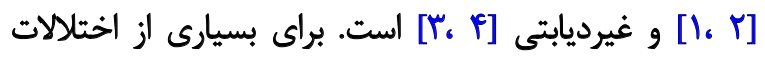

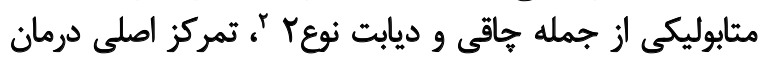

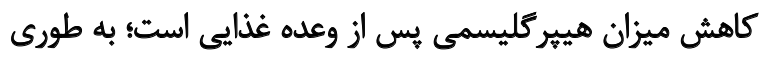

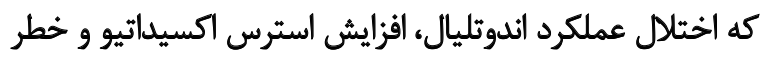

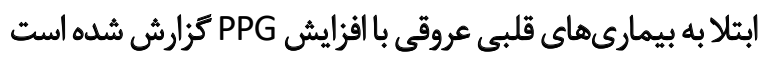

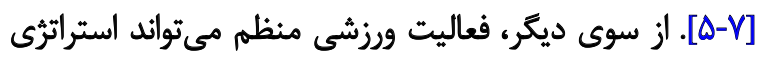

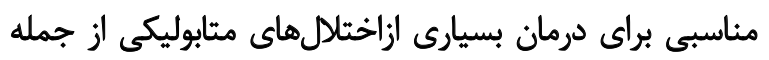

1. Post Prandial Glucose (PPG)

2. Type 2 Diabetes (T2D)

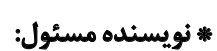

دكتر وازكن ميناسيان

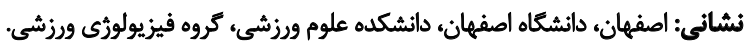
تلفن: يست الكترونيكى: vلr.minasian@spr.ui.ac.ir 
در طول روز صرف نظر از زمان اجرا (ييش از وعده غذايى، هيس از

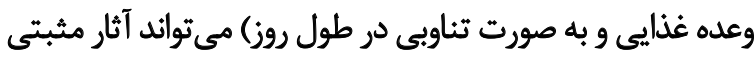

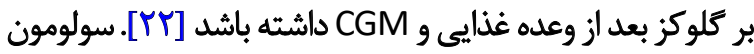

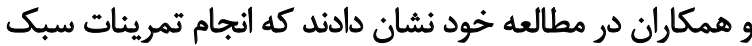

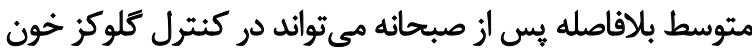

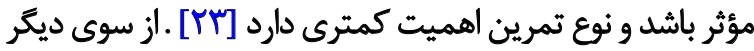

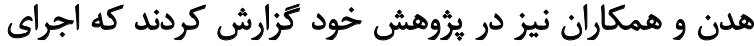

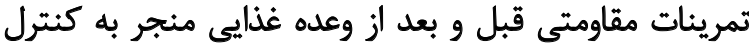

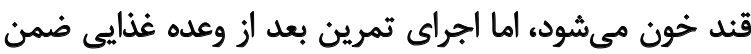

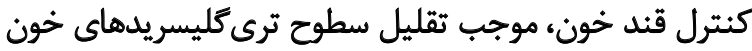

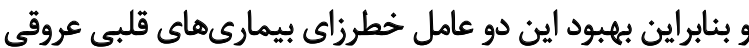

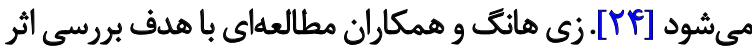

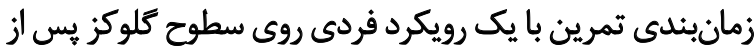

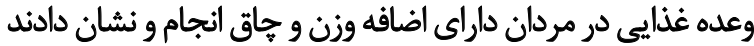

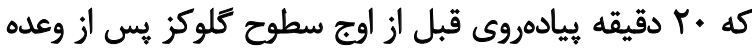

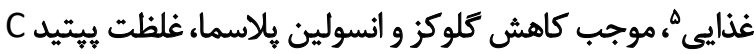

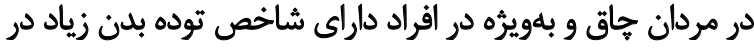

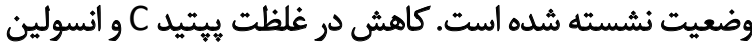

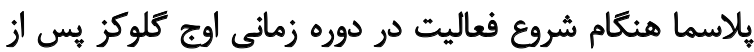

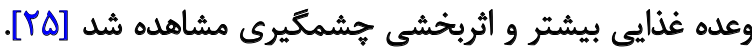

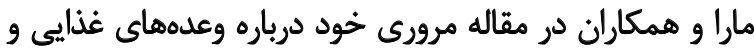

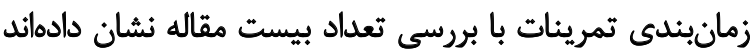

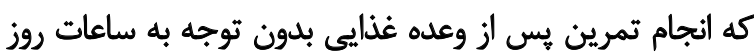

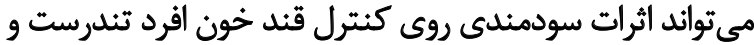

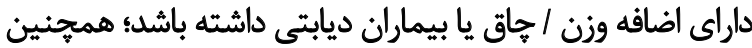

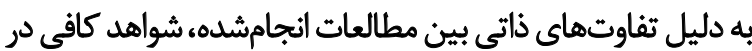

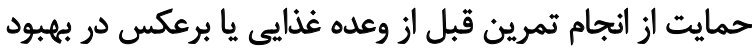

كليسمى وجود ندارد و به مطالعات بيشترى نياز است [ع آن].

از آنجايى كه مطالعات بسيار محدود و متناقضى در اين رابطه

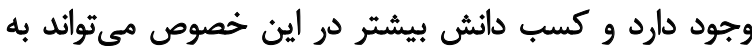

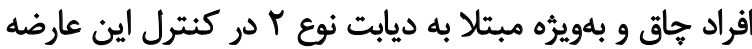

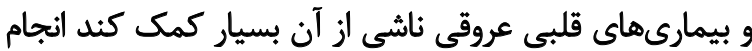

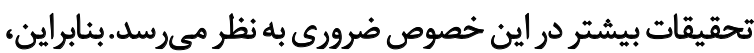

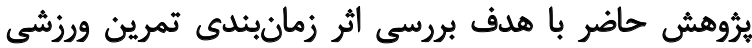

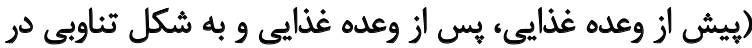

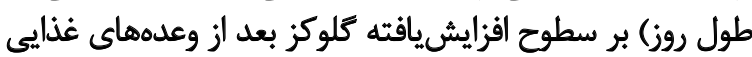
در زنان ميانسال و جاق انجام شد.

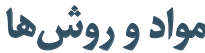

يُروهش حاضر از نوع نيمهتجربى باطرح متقاطع بوده و جامعه

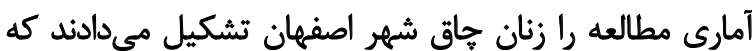
به شكل در دسترس و هدفمند انتخاب شدند. براي اين منظور إنان

5. Postprandial Glucose Peak (PPGP)

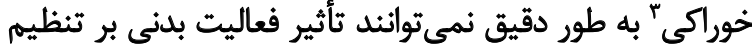

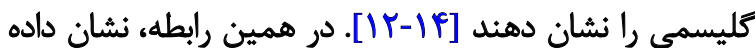

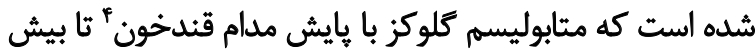

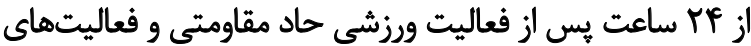

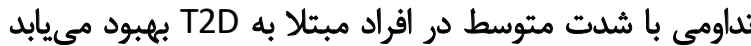

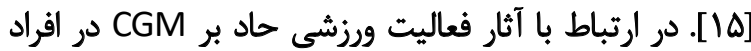

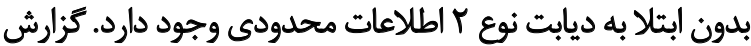

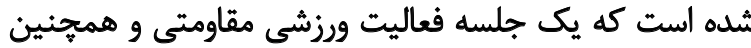

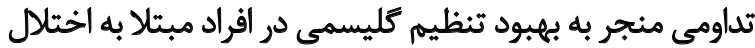

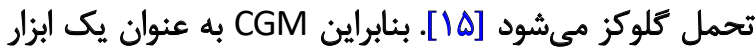

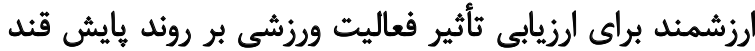

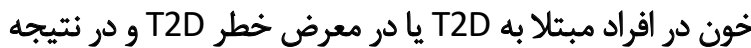

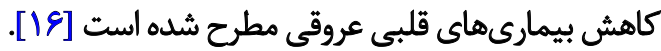

بر اساس مطالعات انجامشده، بيادهروى با شدت كم يا متوسط

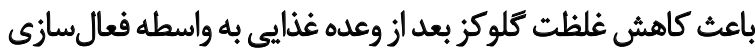

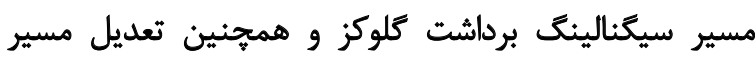

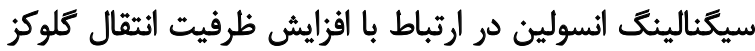

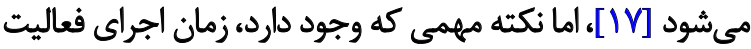

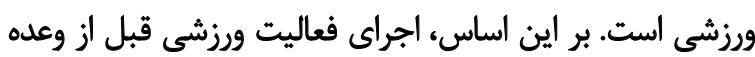

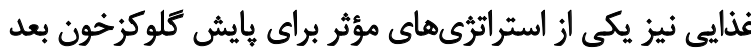

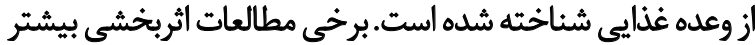

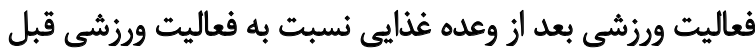

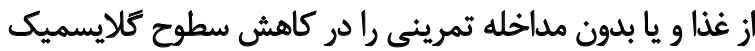

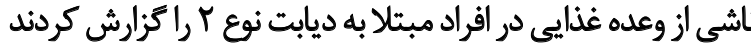

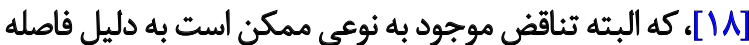

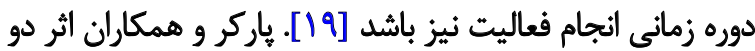

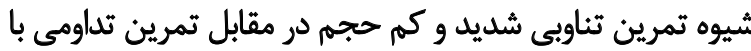

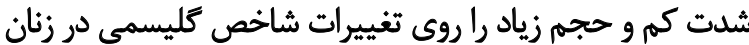

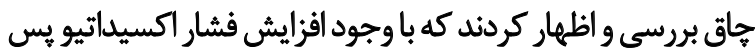

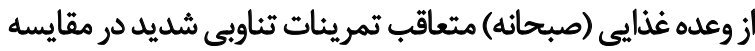

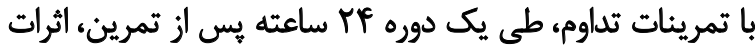

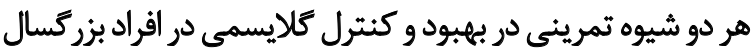

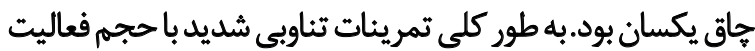

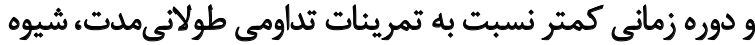

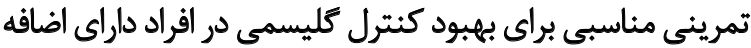

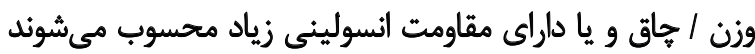

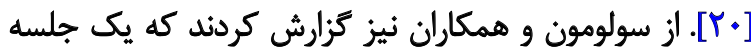

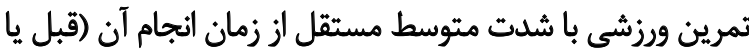

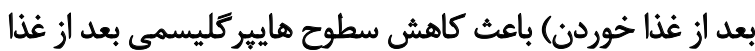

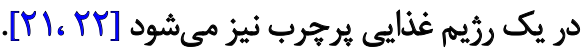
مهمتر اينكه كزارش شده است كه اجراى فعاليت ورزشى حاد

4. Continuous Glucose Monitoring (CGM) 


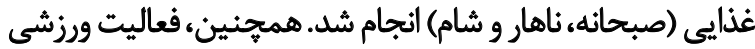

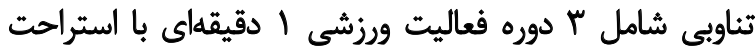

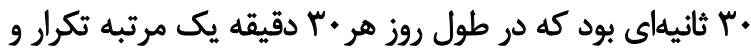

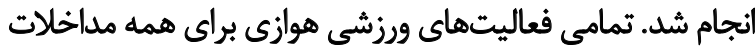

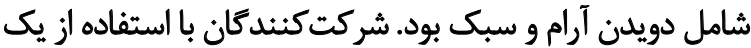

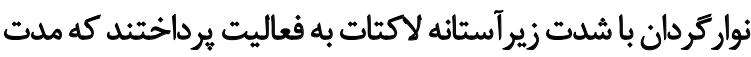
كل آزمون براى تمامى مداخلات درمجموع •. 4 دقيقه بود. هايش مداوم كلوكز: سطوح كلوكز با استفاده از يك دستكاه

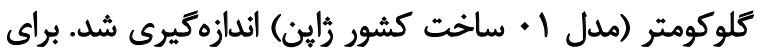

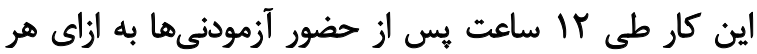

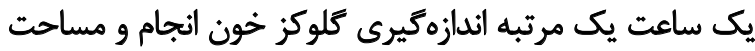
زيرمنحنى (AUC) محاسبه شد.

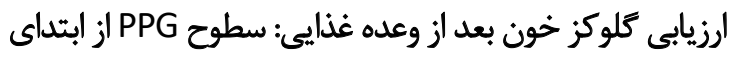

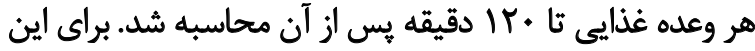

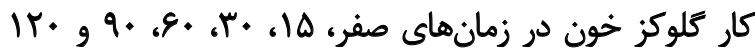

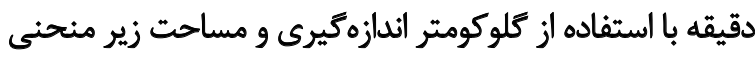
محاسبه شد. (AUC)

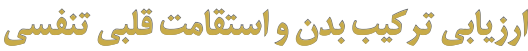

براي اندازهيرى تركيب بلن شامل شاخص توده بلن، درصد

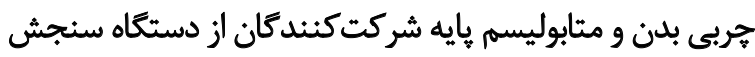

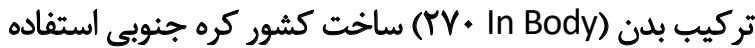

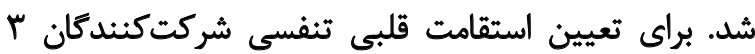

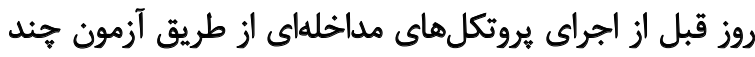

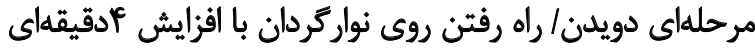

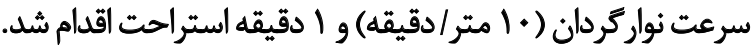

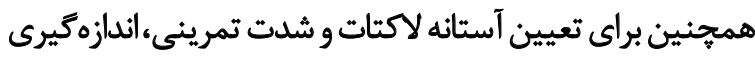

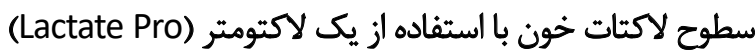

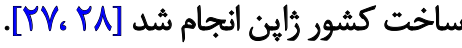

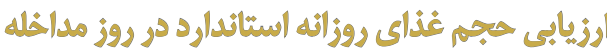

ور ابتدا از شركتكنيدكان درخواست شد تا در دو روز مقادير

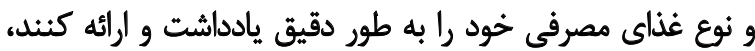

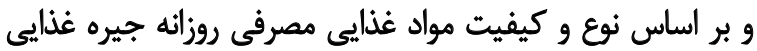

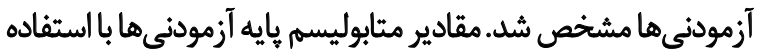

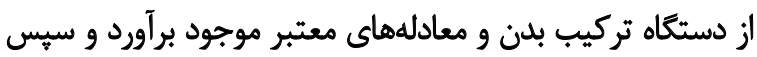

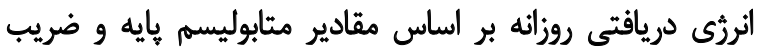

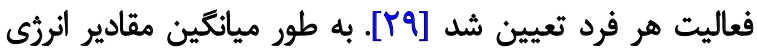

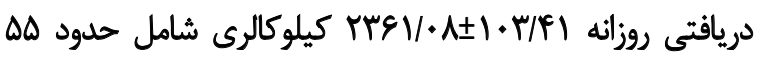

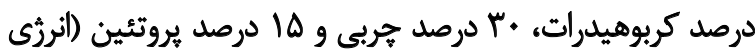

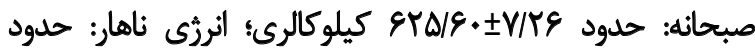

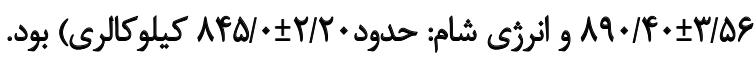

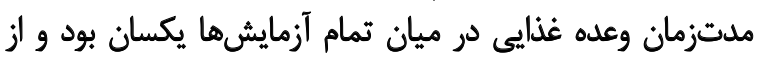

يس از فراخوان اوليه در سطح شهر اصفهان در نهايت · ل نفر

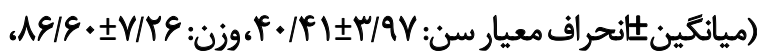

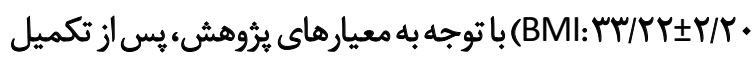

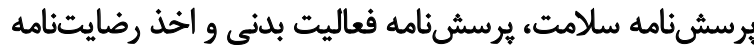

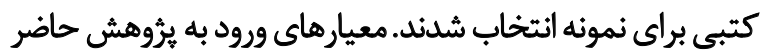

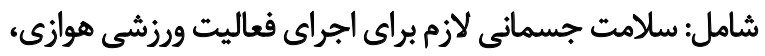

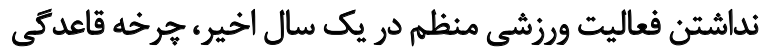

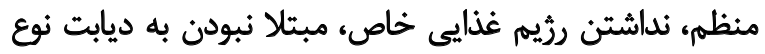

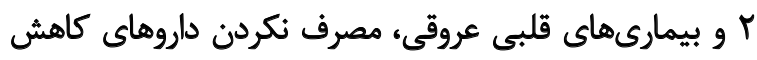

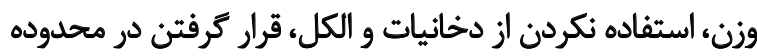

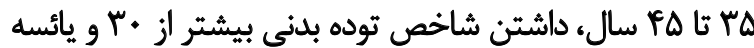

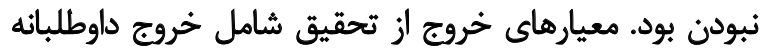

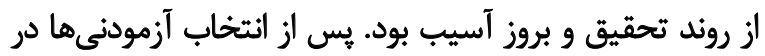

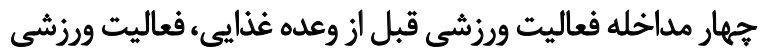

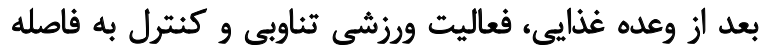

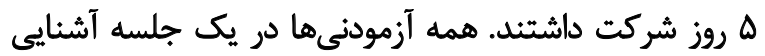

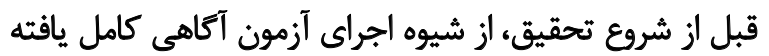

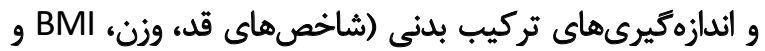

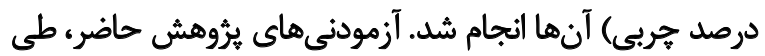

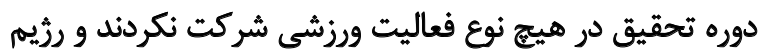

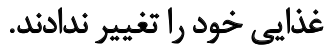

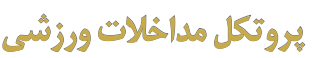

در اين مطالعه مداخلات ورزشى به شكل تصادفى انجام شدند،

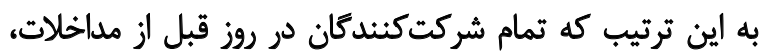

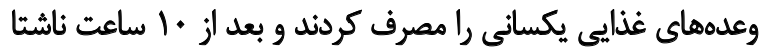

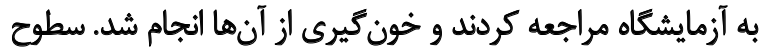

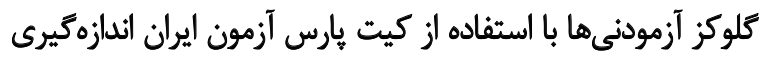

مداخلات در f روز كامل و با ه روز استراحت بين هر جلسه

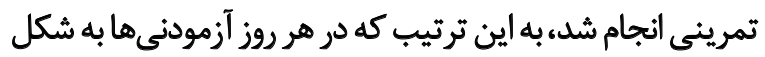

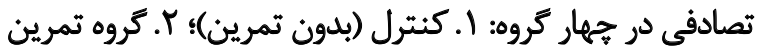

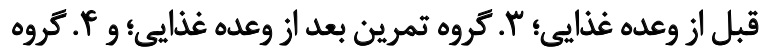

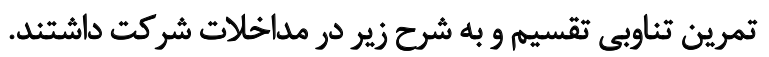

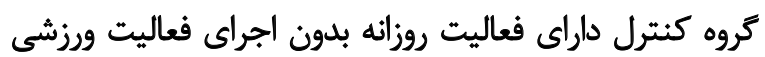

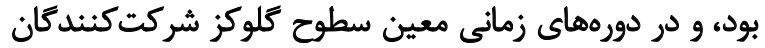
اندازهكيرى شد (تصوير شماره ()).

مداخله فعاليت ورزشى قبل از وعده غذايى شامل • r و وهله

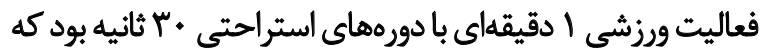

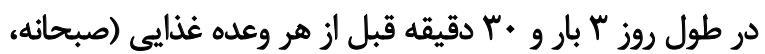

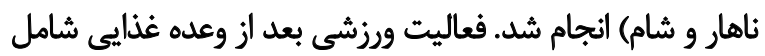

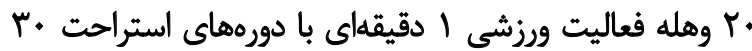

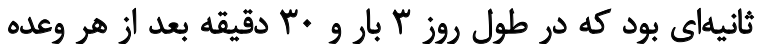




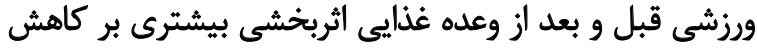

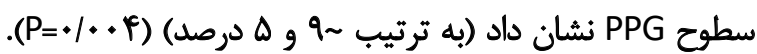

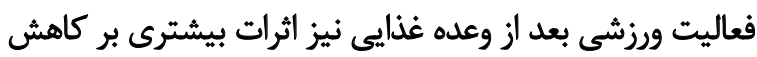

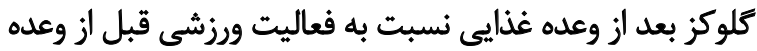

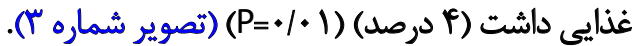

ث

يكى از مهمترين يافتههاى يُروهش حاضر اين بود كه انجام بوري

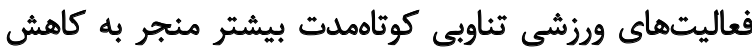

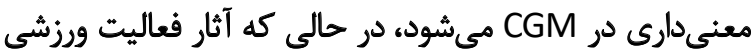

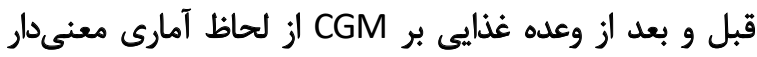

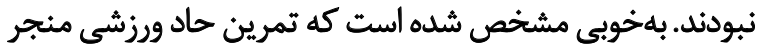

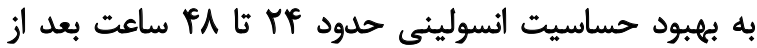

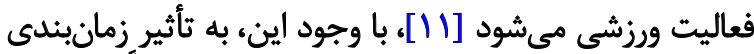

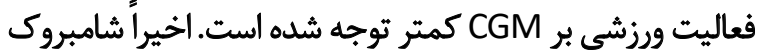

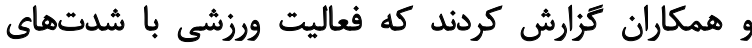

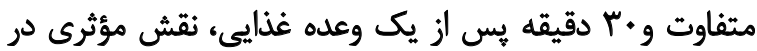

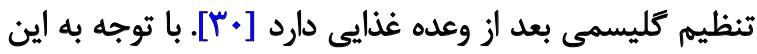

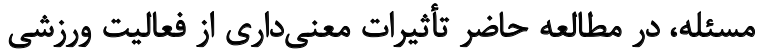

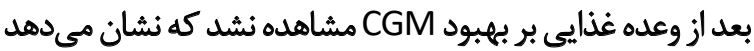

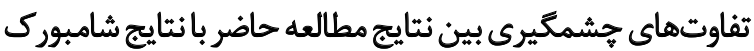

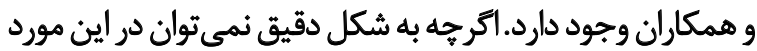

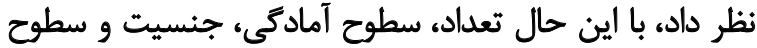

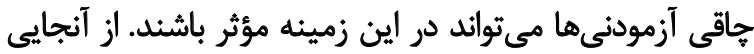

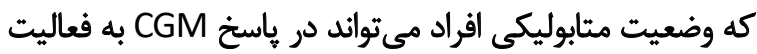

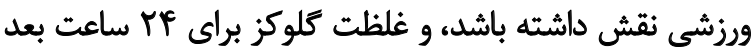

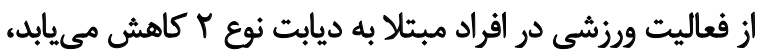

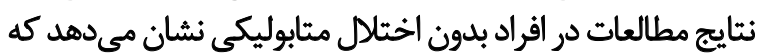

شركت كنيدكان خواسته شد طي مدت ها ه دقيقه غذاى خود را ميل

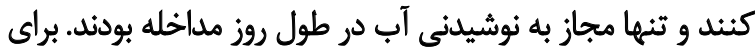

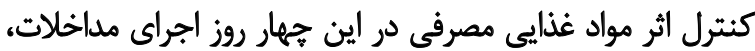

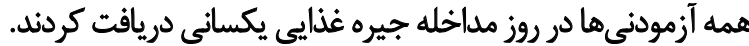
روشهاى آمارى: دادههاى آمارى به شكل ميانكين و انحراف

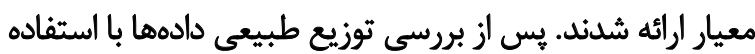

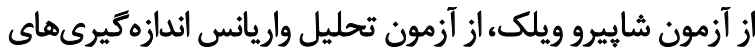

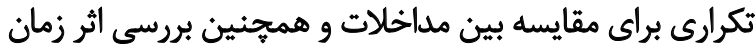

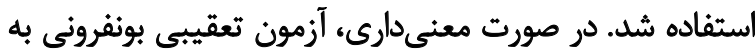

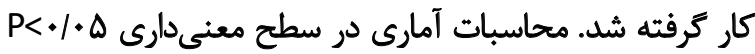

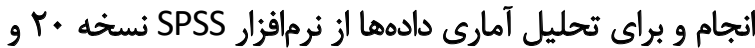

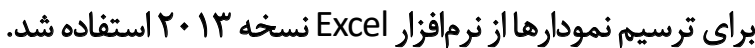

ياقتهها

ويرَكىهاى توصيفى آزمودنىهاى تحقيق در جدول شماره ا رائه شده است.

نتايج تحليل دادها نشان داد كه فعاليتهاى تناوبي كوثامديت

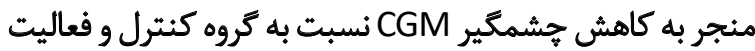

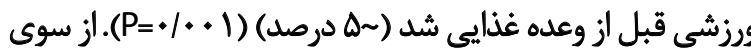

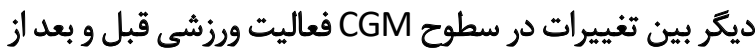

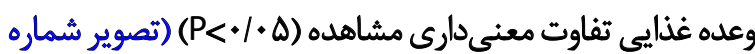

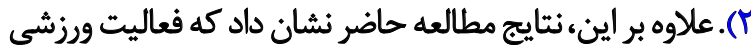

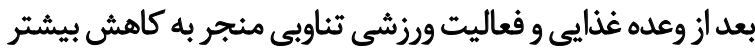

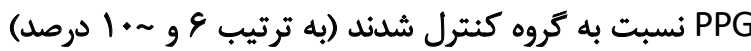

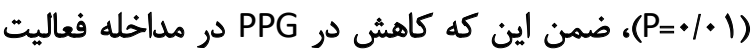

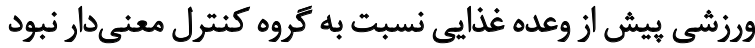

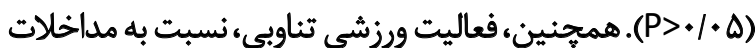

جدول ا. ميانكين و انحراف معيار ويرّكى هاي فردى آزمودنيها.

\begin{tabular}{|c|c|}
\hline ميانكين 土|نحراف معيار & مثيخيرها \\
\hline$f+/ * i \pm r / \cdot q$ & سن (سال) \\
\hline$N \& / E+ \pm V / T E$ & وزن (كيلوكرم) \\
\hline$|\&| / F+ \pm r / \Delta F$ & قد (سائتىمتر) \\
\hline$M T / Y r \pm r / Y$. & شاخص توده بلن (كيلوكرم/متر مربع) \\
\hline$r \Delta / r \cdot \pm r / r \varepsilon$ & درصل جربي \\
\hline $\mid \Delta V=1+\Delta \pm E N 9 F$ & ميزان متابوليسم يايه (كيلوكالرى) \\
\hline$r \Delta / 8 . \pm 1 / \wedge q$ & ظرفيت هوازى (ميلى ليتر/ كيلوكرم در دقيقه) \\
\hline$\Delta / q \cdot \pm \cdot / N^{\prime}$ & سرعت نواركردان (كيلومتر بر ساعت) \\
\hline
\end{tabular}




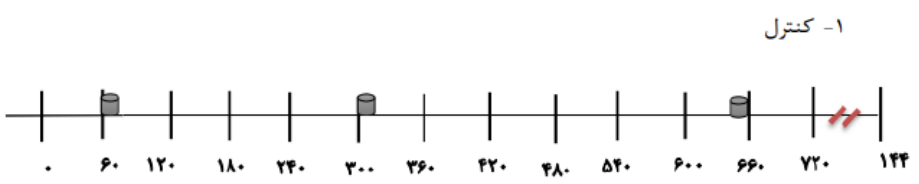

r- قعاليت ورزشى قبل از وعده غذاى

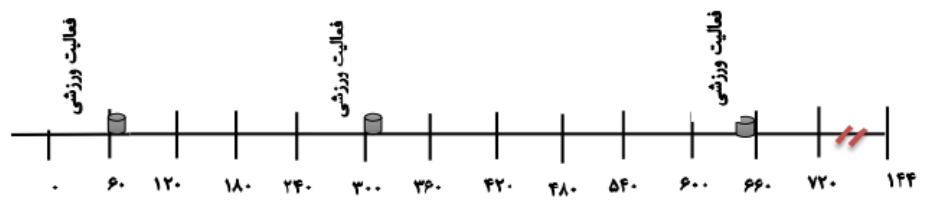

r- فعاليت ورزشى بعد از وعده غذايى

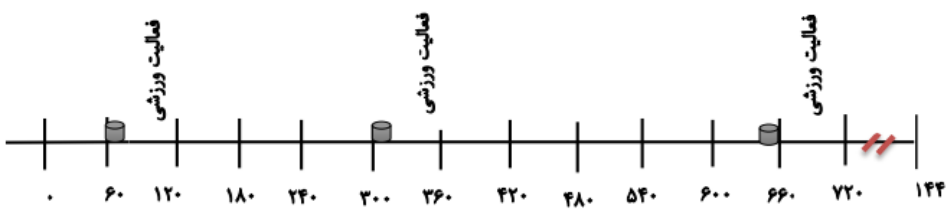

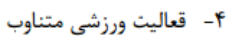

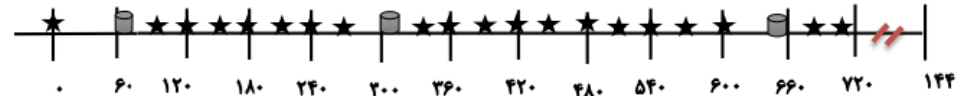

$\sqrt[3]{2}$

تصوير ا. روند انجام مداخلات در تروههاى تحقيق

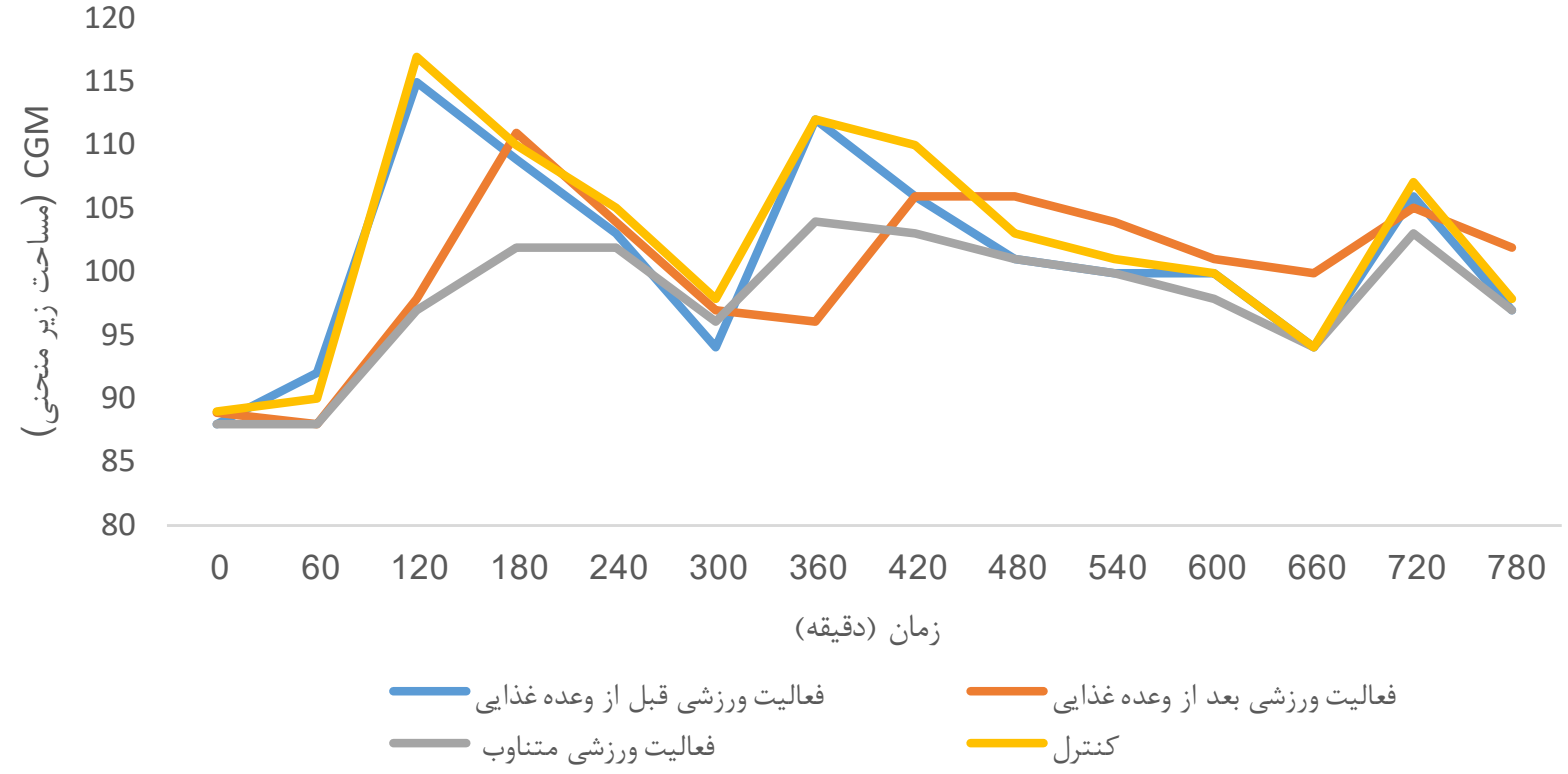




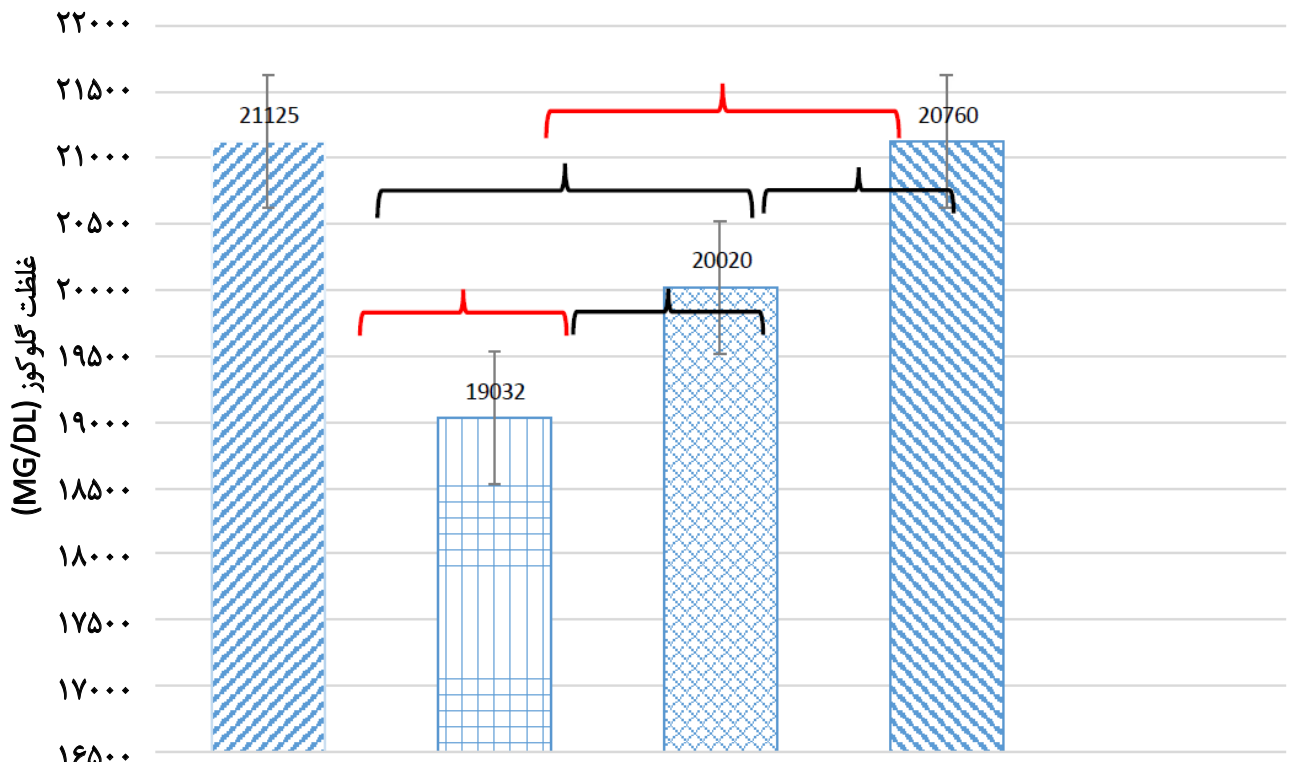

فعاليت ورزشي قبل از وعده فعاليت ورزشى يس از وعده فعاليت تناوبى كوتاه مدت كنترل

غذايى

عذايى

تصوير ب. تغييرات كلوكز يس از وعده غذايى در كروه كوناكون

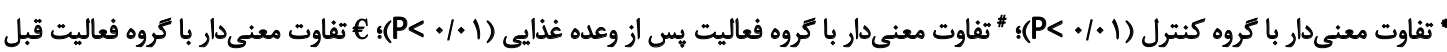

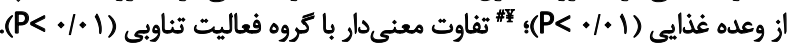

ديابت كاهش مي دهد.از سازوكارهاي مؤثر بر كاهش كلوكز خون

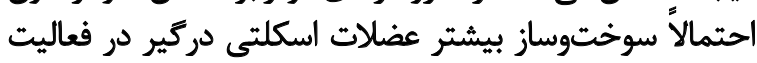

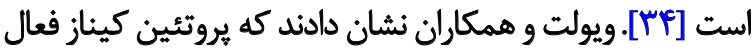
شده با AMP به دليل افزايش نسبت AMP/ATP دور عضله اسكلتى

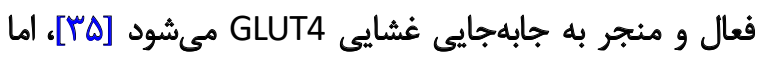

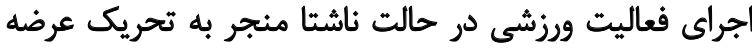

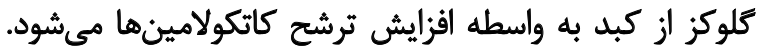

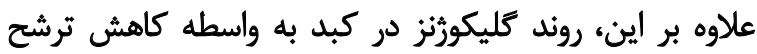

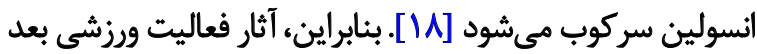

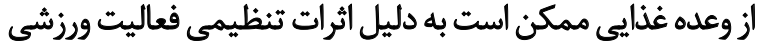

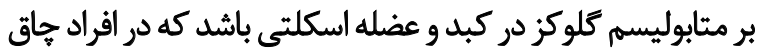

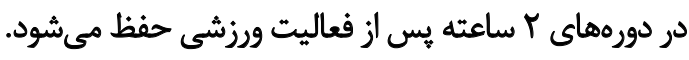

در مقابل، مهمثرين يافتههاى يُؤهش حاضر نشان داد كه

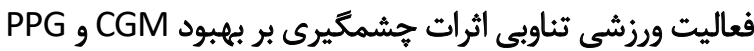

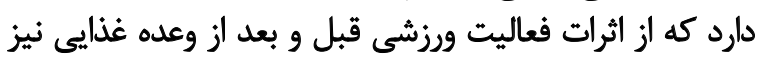

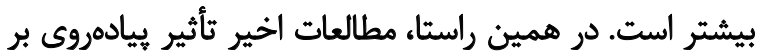

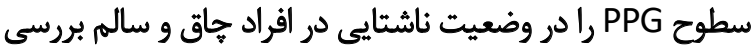

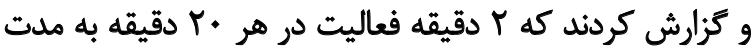

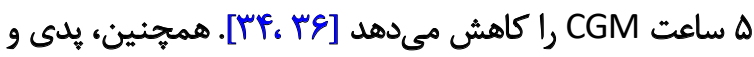

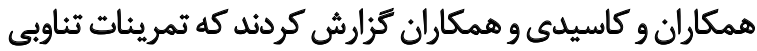

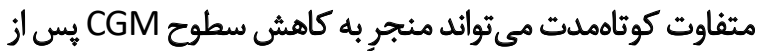

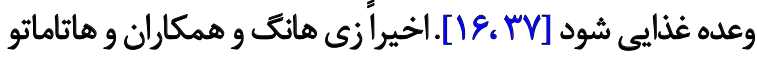
و همكاران در مطالعات خود نشان دادند كه انجام فعاليتهائ
ياسخ كلوكز در مدت كوتاهي (ا تا ب ساعت) حاصل ميشودو.

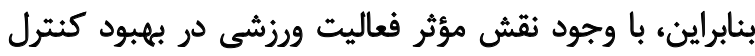

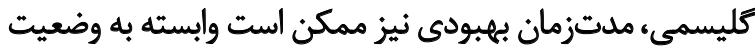

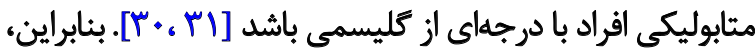

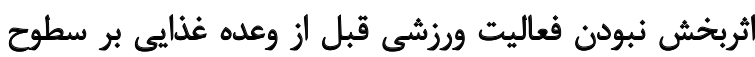

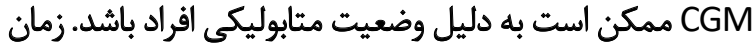

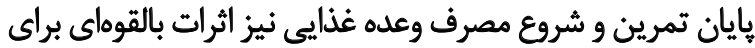

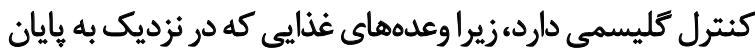
تمرين مصرف شدهاند، واكنش حساسيت انسولينى راني را تعديل

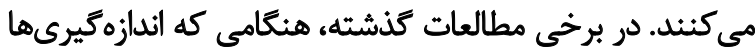

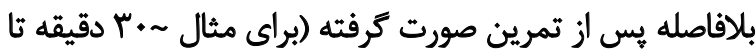

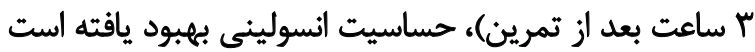

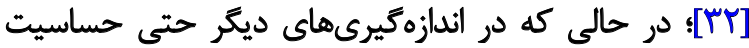

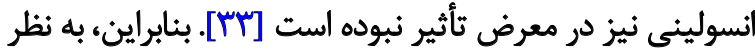

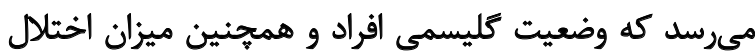

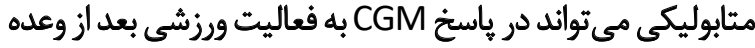

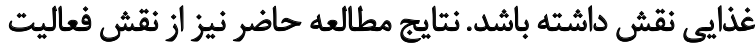

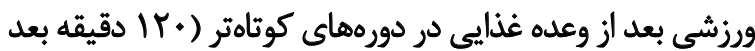
از فعاليت ورزشى) حمايت مى إندا.

از سوى ديكر مشخص شد كه فعاليت ورزشى بعد از وعده

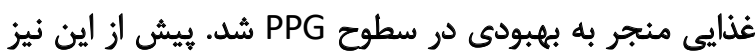

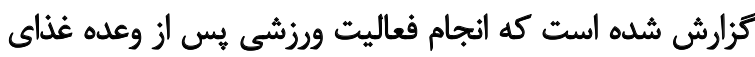

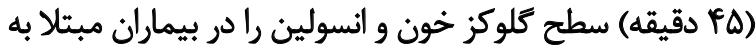




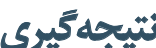

هافتههاى يُروهش حاضر نشان داد كه اجراى فعاليتهاي

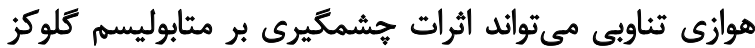

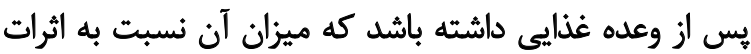

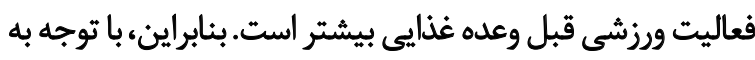

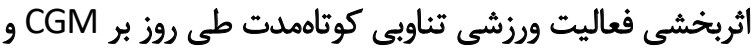

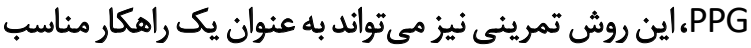

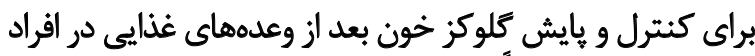

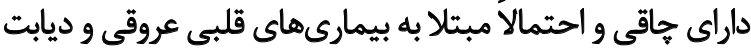
نوع rاستفاده شود.

ملاحظات اخلاقى

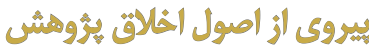

اين مطالعه با اخذ مجوز و دريافت كد اخلاق ئروهشى باشماره

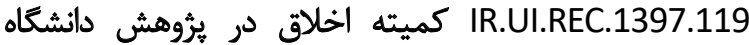

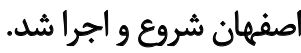

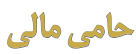

اين مقاله از غاياننامه كارشناسى ارشد نويسنده اول در كروه

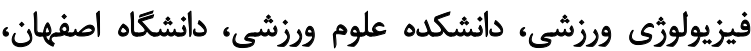

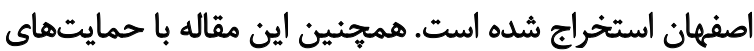

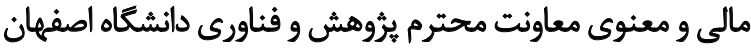

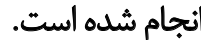

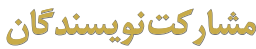

هر دو نويسنده در طراحى، اجرا و نكارش همه بخشهاى ئروهش حاضر مشاركت داشتهاند.

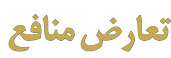

نويسندكان اعلام مى كنئ كه در انتشار اين مقاله هيجگونه

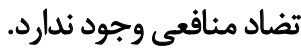

ورزشى تداومى و تناوبى بعد از وعده غذايى مي تواند در كاهش

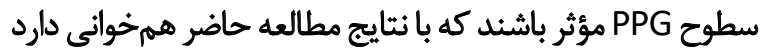

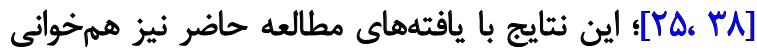

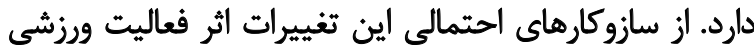

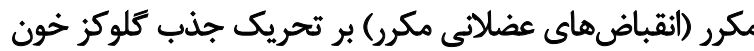

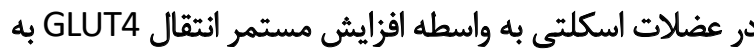

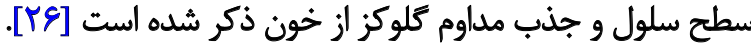

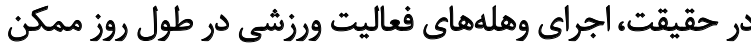

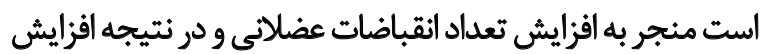

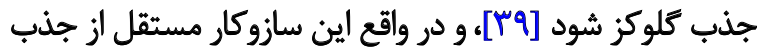

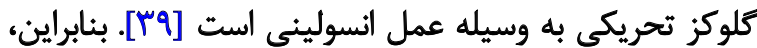

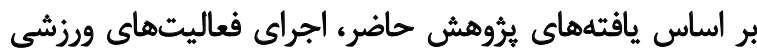

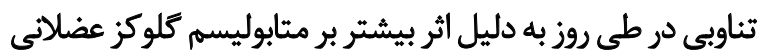

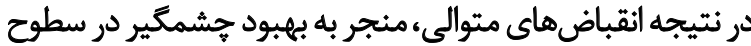
CGM gPG

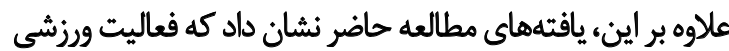

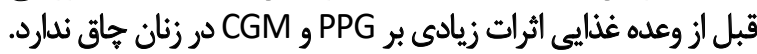

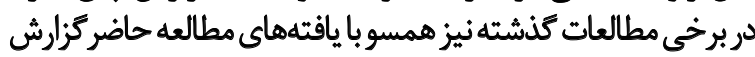

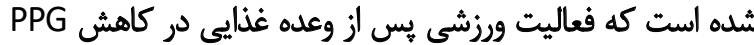

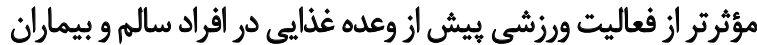

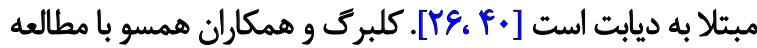

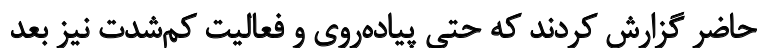
ز زعده غذايى باعث كاهش سطح

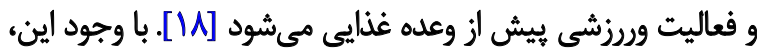

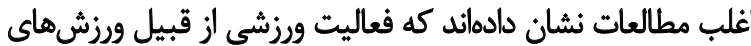

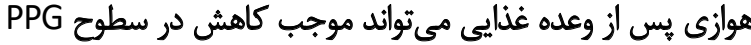

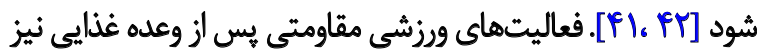

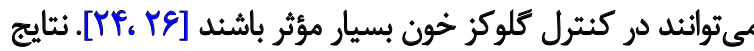

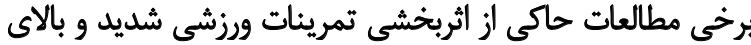

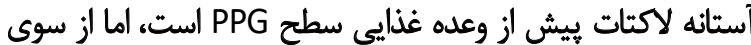

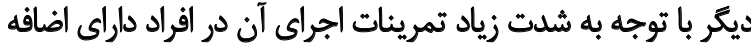

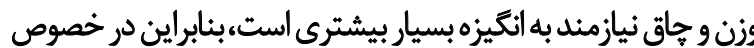

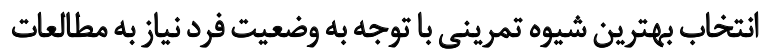

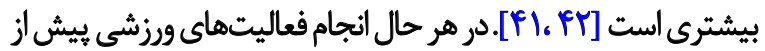

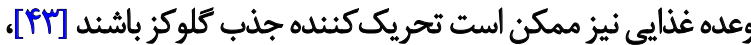

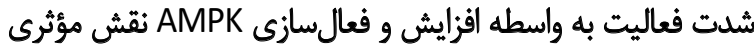

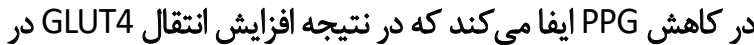

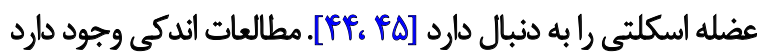

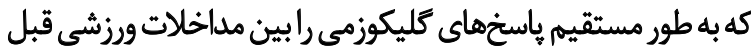

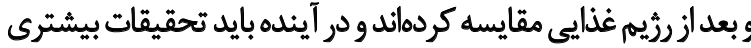

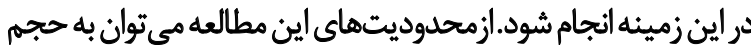

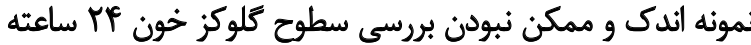

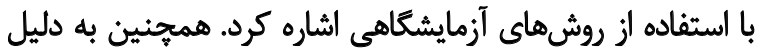

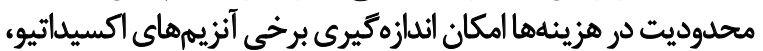

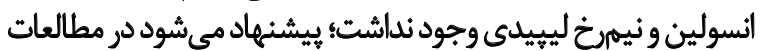

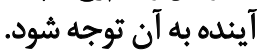




\section{References}

[1] O'Keefe JH, Bell DS. Postprandial hyperglycemia/hyperlipidemia (postprandial dysmetabolism) is a cardiovascular risk factor. Am J Cardiol. 2007; 100(5):899-904. [DOI:10.1016/j.amjcard.2007.03.107] [PMID]

[2] Sorkin JD, Muller DC, Fleg JL, Andres R. The relation of fasting and 2-h postchallenge plasma glucose concentrations to mortality: Data from the Baltimore Longitudinal Study of Aging with a critical review of the literature. Diabetes Care. 2005; 28(11):2626-32. [DOI:10.2337/ diacare.28.11.2626] [PMID]

[3] Lin HJ, Lee BC, Ho YL, Lin YH, Chen CY, Hsu HC, et al. Postprandial glucose improves the risk prediction of cardiovascular death beyond the metabolic syndrome in the nondiabetic population. Diabetes Care. 2009; 32(9):1721-6. [DOI:10.2337/dc08-2337] [PMID] [PMCID]

[4] Ning F, Zhang L, Dekker JM, Onat A, Stehouwer CD, Yudkin JS, et al. DECODE finnish and Swedish study investigators. Development of coronary heart disease and ischemic stroke in relation to fasting and 2-hour plasma glucose levels in the normal range. Cardiovasc Diabetol. 2012; 11:76. [DOI:10.1186/1475-2840-11-76] [PMID] [PMCID]

[5] Cavalot F, Pagliarino A, Valle M, Di Martino L, Bonomo K, Massucco $\mathrm{P}$, et al. Postprandial blood glucose predicts cardiovascular events and all-cause mortality in type 2 diabetes in a 14-year follow-up: Lessons from the San Luigi Gonzaga Diabetes Study. Diabetes Care. 2011; 34(10):2237-43. [DOI:10.2337/dc10-2414] [PMID] [PMCID]

[6] Ceriello A, Taboga C, Tonutti L, Quagliaro L, Piconi L, Bais B, et al. Evidence for an independent and cumulative effect of postprandial hypertriglyceridemia and hyperglycemia on endothelial dysfunction and oxidative stress generation: Effects of short- and longterm simvastatin treatment. Circulation. 2002; 106(10):1211-8. [DOI:10.1161/01.CIR.0000027569.76671.A8] [PMID]

[7] Ceriello A, Esposito K, Piconi L, Ihnat MA, Thorpe JE, Testa R, et al. Oscillating glucose is more deleterious to endothelial function and oxidative stress than mean glucose in normal and type 2 diabetic patients. Diabetes. 2008; 57(5):1349-54. [DOI:10.2337/db08-0063] [PMID]

[8] Tokmakidis SP, Zois CE, Volaklis KA, Kotsa K, Touvra AM. The effects of a combined strength and aerobic exercise program on glucose control and insulin action in women with type 2 diabetes. Eur J Appl Physiol. 2004; 92(4-5):437-42. [DOI:10.1007/s00421-004-1174-6] [PMID]

[9] Azali K, Yavari Y, Hosseinzadeh R, Rahbar Ghazi A. [Effect of aerobic training before and after a meal on serum acylated ghrelin level, daily energy intake and single exercise energy expenditure in overweight men (Persian)]. Metab Exerc. 2019; 9(1):15-25. [DOI:10.22124/ JME.2020.4353]

[10] Bacchi E, Negri C, Trombetta M, Zanolin ME, Lanza M, Bonora E, et al. Differences in the acute effects of aerobic and resistance exercise in subjects with type 2 diabetes: Results from the RAED2 Randomized Trial. PLoS One. 2012; 7(12):e49937. [DOI:10.1371/journal. pone.0049937] [PMID] [PMCID]

[11] Newsom SA, Everett AC, Hinko A, Horowitz JF. A single session of low-intensity exercise is sufficient to enhance insulin sensitivity into the next day in obese adults. Diabetes Care. 2013; 36(9):2516-22. [DOI:10.2337/dc12-2606] [PMID] [PMCID]

[12] Mikus CR, Oberlin DJ, Libla J, Boyle L, Thyfault JP. Glycaemic control is improved by 7 days of aerobic exercise training in patients with type 2 diabetes. Diabetologia. 2012; 55(5):1417-23. [DOI:10.1007/ s00125-012-2490-8] [PMID] [PMCID]
[13] Mikus CR, Oberlin DJ, Libla JL, Taylor AM, Booth FW, Thyfault JP. Lowering physical activity impairs glycemic control in healthy volunteers. Med Sci Sports Exerc. 2012; 44(2):225-31. [DOI:10.1249/ MSS.0b013e31822ac0c0] [PMID] [PMCID]

[14] Mikus CR, Fairfax ST, Libla JL, Boyle L, Vianna LC, Oberlin DJ, et al. 7 days of aerobic exercise training improves conduit artery blood flow following glucose ingestion in patients with type 2 diabetes. J Appl Physiol. 2011; 111(3):657-64. [DOI:10.1152/japplphysiol.00489.2011] [PMID] [PMCID]

[15] van Dijk JW, Manders RJ, Tummers K, Bonomi AG, Stehouwer CD, Hartgens F, et al. Both resistance- and endurance-type exercise reduce the prevalence of hyperglycaemia in individuals with impaired glucose tolerance and in insulin-treated and non-insulin-treated type 2 diabetic patients. Diabetologia. 2012; 55(5):1273-82. [DOI:10.1007/s00125-011 2380-5] [PMID] [PMCID]

[16] Cassidy S, Thoma C, Houghton D, Trenell MI. High-intensity interval training: A review of its impact on glucose control and cardiometabolic health. Diabetologia. 2017; 60(1):7-23. [DOI:10.1007/s00125-016-41061] [PMID] [PMCID]

[17] Bergouignan A, Latouche C, Heywood S, Grace MS, Reddy-Luthmoodoo M, Natoli AK, et al. Frequent interruptions of sedentary time modulates contraction- and insulin-stimulated glucose uptake pathways in muscle: Ancillary analysis from randomized clinical trials. Sci Rep. 2016 6:32044. [DOI:10.1038/srep32044] [PMID] [PMCID]

[18] Colberg SR, Zarrabi L, Bennington L, Nakave A, Thomas Somma C, Swain DP, et al. Postprandial walking is better for lowering the glycemic effect of dinner than pre-dinner exercise in type 2 diabetic individuals. J Am Med Dir Assoc. 2009; 10(6):394-7. [DOI:10.1016/j.jamda.2009.03.015] [PMID]

[19] Terada T, Wilson BJ, Myette-Côté E, Kuzik N, Bell GJ, McCargar L, et al. Targeting specific interstitial glycemic parameters with high-intensity interval exercise and fasted-state exercise in type 2 diabetes. Metabolism. 2016; 65(5):599-608. [DOI:10.1016/j.metabol.2016.01.003] [PMID]

[20] Parker L, Shaw CS, Banting L, Levinger I, Hill KM, McAinch AJ, et al. Acute low-volume high-intensity interval exercise and continuous moderate-intensity exercise elicit a similar improvement in 24-h glycemic control in overweight and obese adults. Front Physiol. 2017; 7:661. [DOI:10.3389/fphys.2016.00661] [PMID] [PMCID]

[21] Katsanos CS, Moffatt RJ. Acute effects of premeal versus postmeal exercise on postprandial hypertriglyceridemia. Clin J Sport Med. 2004 14(1):33-9. [DOI:10.1097/00042752-200401000-00006] [PMID]

[22] Solomon TPJ. Sources of inter-individual variability in the therapeutic response of blood glucose control to exercise in type 2 diabetes: Going beyond exercise dose. Front Physiol. 2018; 9:896. [DOI:10.3389/ fphys.2018.00896] [PMID] [PMCID]

[23] Solomon TPJ, Tarry E, Hudson CO, Fitt Al, Laye MJ. Immediate postbreakfast physical activity improves interstitial postprandial glycemia: A comparison of different activity-meal timings. Pflugers Arch. 2020 472(2):271-80. [DOI:10.1007/s00424-019-02300-4] [PMID] [PMCID]

[24] Heden TD, Winn NC, Mari A, Booth FW, Rector RS, Thyfault JP, et al. Postdinner resistance exercise improves postprandial risk factors more effectively than predinner resistance exercise in patients with type 2 diabetes. J Appl Physiol (1985). 2015; 118(5):624-34. [DOI:10.1152/japplphysiol.00917.2014] [PMID] [PMCID]

[25] Zhang X, Wongpipit W, Sun F, Sheridan S, Huang WYJ, Sit CHP, et al. Walking initiated 20 minutes before the time of individual postprandial glucose peak reduces the glucose response in young men with overweight or obesity: A randomized crossover study. J Nutr. 2021 151(4):866-75. [DOI:10.1093/jn/nxaa420] [PMID] 
[26] Aqeel M, Forster A, Richards EA, Hennessy E, McGowan B, Bhadra $A$, et al. The effect of timing of exercise and eating on postprandial response in adults: A systematic review. Nutrients. 2020; 12(1):221. [DOI:10.3390/nu12010221] [PMID] [PMCID]

[27] Wicks JR, Oldridge N B. How accurate is the prediction of maximal oxygen uptake with treadmill testing? PLoS One. 2016; 11(11):e0166608. [DOI:10.1371/journal.pone.0166608] [PMID] [PMCID]

[28] Christle JW, Arena R. Cardiopulmonary exercise testing and prescription of exercise. In: Pressler A, Niebauer J, editors. Textbook of Sports and exercise cardiology. Germany: Springer, Cham; 2020. [DOI:10.1007/978-3-030-35374-2_43]

[29] Xue J, Li S, Zhang Y, Hong P. Accuracy of predictive resting-metabolic-rate equations in Chinese mainland adults. Int J Environ Res Public Health. 2019; 16(15):2747. [DOI:10.3390/ijerph16152747] [PMID] [PMCID]

[30] Shambrook P, Kingsley MI, Wundersitz DW, Xanthos PD, Wyckelsma VL, Gordon BA. Glucose response to exercise in the post-prandial period is independent of exercise intensity. Scand J Med Sci Sports. 2018; 28(3):939-46. [DOI:10.1111/sms.12999] [PMID]

[31] Manders RJ, Van Dijk JW, van Loon L. Low-intensity exercise reduces the prevalence of hyperglycemia in type 2 diabetes. Med Sci Sports Exerc. 2010; 42(2):219-25. [DOI:10.1249/MSS.0b013e3181b3b16d] [PMID]

[32] Holtz KA, Stephens BR, Sharoff CG, Chipkin SR, Braun B. The effect of carbohydrate availability following exercise on whole-body insulin action. Appl Physiol Nutr Metab. 2008; 33(5):946-56. [DOI:10.1139/ H08-077] [PMID]

[33] Weltman NY, Rynders CA, Gaesser G, Weltman JY, Barrett EJ, Arthur W. Exercise intensity does not affect glucose disposal in euglycemic abdominally obese adults. Obe Metab. 2010; 6(4):86-93. https://asu. pure.elsevier.com/en/publications/exercise-intensity-does-not-affectglucose-disposal-in-euglycemic

[34] Larsen JJ, Dela F, Kjaer M, Galbo H. The effect of moderate exercise on postprandial glucose homeostasis in NIDDM patients. Diabetologia. 1997; 40(4):447-53. [DOI:10.1007/s001250050699] [PMID]

[35] Viollet B, Lantier L, Devin-Leclerc J, Hebrard S, Amouyal C, Mounier $\mathrm{R}$, et al. Targeting the AMPK pathway for the treatment of type 2 diabetes. Front Biosci (Landmark Ed). 2009; 14:3380-400. [DOI:10.2741/3460] [PMID]

[36] Bailey DP, Locke CD. Breaking up prolonged sitting with lightintensity walking improves postprandial glycemia, but breaking up sitting with standing does not. J Sci Med Sport. 2015; 18(3):294-8. [DOI:10.1016/j.jsams.2014.03.008] [PMID]

[37] Peddie MC, Bone JL, Rehrer NJ, Skeaff CM, Gray AR, Perry TL. Breaking prolonged sitting reduces postprandial glycemia in healthy, normal-weight adults: A randomized crossover trial. Am J Clin Nutr. 2013; 98(2):358-66. [DOI:10.3945/ajcn.112.051763] [PMID]

[38] Hatamoto Y, Goya R, Yamada Y, Yoshimura E, Nishimura S, Higaki $\mathrm{Y}$, et al. Effect of exercise timing on elevated postprandial glucose levels. J Appl Physiol (1985). 2017; 123(2):278-84. [DOI:10.1152/japplphysiol.00608.2016] [PMID]

[39] Sylow L, Kleinert M, Richter EA, Jensen TE. Exercise-stimulated glucose uptake- regulation and implications for glycaemic control. Nat Rev Endocrinol. 2017; 13(3):133-48. [DOI:10.1038/nrendo.2016.162] [PMID]
[40] Haxhi J, Scotto di Palumbo A, Sacchetti M. Exercising for metabolic control: Is timing important? Ann Nutr Metab. 2013; 62(1):14-25. [DOI:10.1159/000343788] [PMID]

[41] Francois ME, Baldi JC, Manning PJ, Lucas SJ, Hawley JA, Williams MJ, et al. 'Exercise snacks' before meals: A novel strategy to improve glycaemic control in individuals with insulin resistance. Diabetologia. 2014; 57(7):1437-45. [DOI:10.1007/s00125-014-3244-6] [PMID]

[42] Rynders CA, Weltman JY, Jiang B, Breton M, Patrie J, Barrett EJ, et al. Effects of exercise intensity on postprandial improvement in glucose disposal and insulin sensitivity in prediabetic adults. J Clin Endocrinol Metab. 2014; 99(1):220-8. [DOI:10.1210/jc.2013-2687] [PMID] [PMCID]

[43] Garetto LP, Richter EA, Goodman MN, Ruderman NB. Enhanced muscle glucose metabolism after exercise in the rat: The two phases. Am J Physiol. 1984; 246(6 Pt 1):E471-5. [DOI:10.1152/ ajpendo.1984.246.6.E471] [PMID]

[44] Chen ZP, Stephens TJ, Murthy S, Canny BJ, Hargreaves M, Witters $\mathrm{LA}$, et al. Effect of exercise intensity on skeletal muscle AMPK signaling in humans. Diabetes. 2003; 52(9):2205-12. [DOI:10.2337/diabetes.52.9.2205] [PMID]

[45] Fujii N, Hayashi T, Hirshman MF, Smith JT, Habinowski SA, Kaijser L, et al. Exercise induces isoform-specific increase in 5'AMP-activated protein kinase activity in human skeletal muscle. Biochem Biophys Res Commun. 2000; 273(3):1150-5. [DOI:10.1006/bbrc.2000.3073] [PMID] 